\title{
640 Gbit/s OTDM Lab-Transmission and 320 Gbit/s Field- Transmission with SOA-based Clock Recovery
}

\author{
H.C. Hansen Mulvad ${ }^{(*)}$, E. Tangdiongga, O. Raz, J. Herrera, H. de Waardt, and H.J.S Dorren \\ COBRA Research Institute, Eindhoven University of Technology, PT-12, P.O. Box 513, NL-5600 MB Eindhoven, The Netherlands \\ (*) on leave from: COM•DTU, Technical University of Denmark, Building 343, DK-2800 Kgs. Lyngby, Denmark \\ E-mail:hchm@com.dtu.dk
}

\begin{abstract}
We demonstrate low-jitter 40GHz baserate clock recovery from 640Gbit/s OTDM-data after 50km labtransmission, using a potentially integrable injection-locked loop circuit with SOA-based phase-comparison at low data-powers. A $54.3 \mathrm{~km}$ field-transmission test at $320 \mathrm{Gbit} / \mathrm{s}$ is also performed.
\end{abstract}

(C)2008 Optical Society of America

OCIS codes: (060.2330) Fiber optics communications; (060.4510) Optical communications.

\section{Introduction}

Clock recovery (CR) is the essential functionality which allows a receiver to synchronize to a transmitted data signal in an optical communication system. In a network based on optical time-division multiplexed (OTDM) data signals, the CR circuit must extract a clock at the baserate, which is the repetition rate of the individual data channels constituent of the OTDM signal. This requires an optical phase comparison between the received OTDM signal and the locally generated clock signal. Such a phase comparator must have a very high timing-resolution, i.e. lower than the bit-slot duration which in the case of a $640 \mathrm{Gbit} / \mathrm{s}$ OTDM signal is $\sim 1.5 \mathrm{ps}$. Earlier demonstrations of clock recovery from high-speed data signals have exploited a.o. four-wave mixing in a laser-diode amplifier at $400 \mathrm{Gbit} / \mathrm{s}$ [1], electroabsorption at $320 \mathrm{Gbit} / \mathrm{s}$ [2], and filtered chirp from an SOA at $320 \mathrm{Gbit} / \mathrm{s}$ [3]. It has been demonstrated that a semiconductor optical amplifier (SOA) can exhibit a time-response faster than $\sim 1$ ps when it is assisted by filtered chirp, which has been used for demultiplexing from 640 Gbit/s [4]. The advantages of an SOA is further that it can be integrated, its stability, and that it requires low optical power for switching.

In this paper, we perform $50 \mathrm{~km}$ lab-transmission of a $640 \mathrm{Gbit} / \mathrm{s}$ on-off keyed (OOK) OTDM data signal and subsequent clock recovery of the $40 \mathrm{GHz}$ baserate clock by using a potentially integrable clock recovery circuit. It is an injection-locked loop containing an SOA as optical phase comparator, a Gunn oscillator, and a pulse source. Phase-noise measurements on the electrical clock from the Gunn oscillator result in very low timing jitter values, both for short and long PRBS data patterns, and for low data input powers to the SOA (down to $-6 \mathrm{dBm}$ ). To further test the robustness of the CR circuit against phase perturbations, it is used after transmission over a $54.3 \mathrm{~km}$ field-installed SMF fibre link. Due to imperfect dispersion compensation of the link, the bit-rate had to be lowered to $320 \mathrm{Gbit} / \mathrm{s}$. This test also results in a successful recovery of the $40 \mathrm{GHz}$ baserate clock with low jitter-values (<200 fs).

\section{Operation principle and experimental set-up}

The experimental set-up is shown in Fig. 1, which covers both the 640 Gbit/s lab-trial and the 320 Gbit/s field-trial.

The $640 \mathrm{Gbit} / \mathrm{s}$ data signal consists of 16 bit-interleaved $40 \mathrm{Gbit} / \mathrm{s}$ OOK-modulated channels, all at the same wavelength

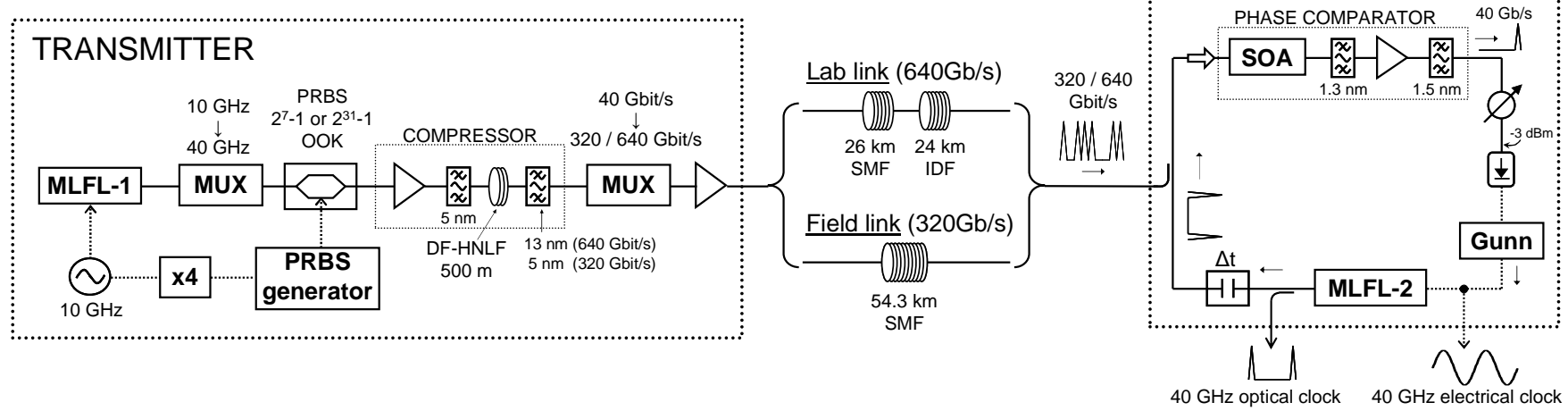

Fig. 1. Experimental set-up for the $640 \mathrm{Gbit} / \mathrm{s}$ lab-trial and the $320 \mathrm{Gbit} / \mathrm{s}$ field-trial. 


\section{OWS2.pdf}

and in the same polarization. It is generated as follows. A Mode-Locked Fibre Ring Laser (MLFL-1) at $\lambda_{1}=1560 \mathrm{~nm}$ emits $\sim 2$ ps pulses at $10 \mathrm{GHz}$. These are then multiplexed up to $40 \mathrm{GHz}$ by a passive fibre delay-line multiplexer (MUX). The $40 \mathrm{GHz}$ pulses are OOK-modulated to $40 \mathrm{Gbit} / \mathrm{s}$ with a PRBS pattern, and then injected to a pulse compression stage. This is based on self-phase modulation (SPM) induced spectral broadening in a $500 \mathrm{~m}$ dispersion-flattened highly non-linear fibre (DF-HNLF) with a dispersion D=-0.36 ps/nm $\mathrm{km}$ and slope $0.004 \mathrm{ps} / \mathrm{nm}^{2} \cdot \mathrm{km}$ at $1560 \mathrm{~nm}$. The output of the DF-HNLF is offcarrier filtered with a $13 \mathrm{~nm}$ bandpass filter (BPF) centered at $1554 \mathrm{~nm} \mathrm{[5],} \mathrm{as} \mathrm{can} \mathrm{be} \mathrm{seen} \mathrm{in} \mathrm{Fig.} 2$ (a). The SPM in the DFHNLF results in an up-chirping of the pulses, which are then compressed by the positive dispersion in the remainder of the transmitter, where the $40 \mathrm{Gbit} / \mathrm{s}$ pulses are multiplexed up to $640 \mathrm{Gbit} / \mathrm{s}$ by a $2^{7}-1$ PRBS-maintaining MUX. At the output of the transmitter, the pulses are thus compressed to $\sim 700 \mathrm{fs}$ with low pedestals. The $640 \mathrm{Gbit} / \mathrm{s}$ data are then transmitted through a $50 \mathrm{~km}$ dispersion-managed laboratory fibre link consisting of $26 \mathrm{~km}$ standard single-mode fibre (SMF) and $24 \mathrm{~km}$ inversedispersion fibre (IDF). The $640 \mathrm{Gbit} / \mathrm{s}$ data before and after transmission are shown in Fig. 2 (b) and (c), respectively.
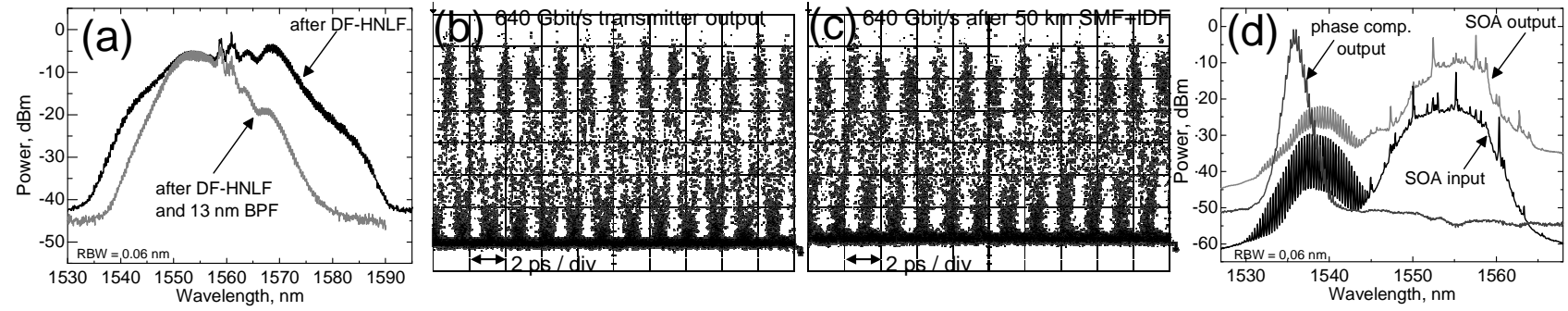

Fig. 2. $640 \mathrm{Gbit} / \mathrm{s}$ lab-trial. (a) $40 \mathrm{Gbit} / \mathrm{s}$ spectra in the compression stage, (b) $640 \mathrm{Gbit} / \mathrm{s}$ data pulses at the transmitter output, measured by a $700 \mathrm{GHz}$ sampling scope, (c) $640 \mathrm{Gbit} / \mathrm{s}$ after $26 \mathrm{~km} \mathrm{SMF}+24 \mathrm{~km} \mathrm{IDF}$ at the input to the SOA, (d) Spectra of the input/output of the SOA showing the $640 \mathrm{Gbit} / \mathrm{s}$ data and $40 \mathrm{GHz}$ probe, and spectrum of the demultiplexed $40 \mathrm{Gbit} / \mathrm{s}$ after the BPFs (phase comparator output).

The clock recovery circuit, shown in Fig. 1, is an optical injection-locked loop containing an SOA as phase comparator, a $40 \mathrm{GHz}$ mode-locked fibre ring laser (MLFL-2) emitting $<1 \mathrm{ps}$ pulses at $\lambda_{2}=1538 \mathrm{~nm}$, and a Gunn oscillator in injectionlocked mode. The SOA is designed with a high optical confinement for optical signal processing, and has the following characteristics: length $1.9 \mathrm{~mm}$, polarization dependence 2-3 dB, small signal gain 13-14 dB, saturation output power $12 \mathrm{dBm}$, recovery time $\sim 8 \mathrm{ps}$, and it is biased to $400 \mathrm{~mA}$. The $640 \mathrm{Gbit} / \mathrm{s}$ data is coupled into the loop and injected to the SOA with an average power of only $0 \mathrm{dBm}$, together with the $40 \mathrm{GHz}$ probe pulses from the MLFL-2 at $-14 \mathrm{dBm}$. The data acts as a pump and modulates the SOA gain, which results in a chirping of the probe pulses. This chirp is extracted by two narrow BPFs, centered at $1536 \mathrm{~nm}$, resulting in a $40 \mathrm{Gbit} / \mathrm{s}$ inverted copy of the baserate channel data onto the probe pulse train [4]. This $40 \mathrm{Gbit} / \mathrm{s}$ demultiplexed data signal is detected by a $43 \mathrm{GHz}$ photodiode followed by a transimpedance amplifier. The resulting electrical $40 \mathrm{Gbit} / \mathrm{s}$ is injected into the Gunn oscillator which is in injection-locked operation. The free-running resonance frequency of the Gunn cavity is mechanically tunable, and a DC supply to the Gunn diode allows for fine-tuning. When injected with a $40 \mathrm{Gbit} / \mathrm{s}$ signal, the Gunn oscillator emits a high-quality electrical sine at $40 \mathrm{GHz}$. This is used to synchronize the MLFL-2 via an internal phase-locked loop. The CR loop length is adjusted by a variable optical time-delay $\Delta t$, in order to fulfill the phase-matching condition for the $40 \mathrm{GHz}$ baserate of the incoming OTDM data signal.

\section{Results}

The $40 \mathrm{GHz}$ clock is successfully recovered with low jitter from the $640 \mathrm{Gbit} / \mathrm{s}$ data after the $50 \mathrm{~km}$ SMF+IDF span.
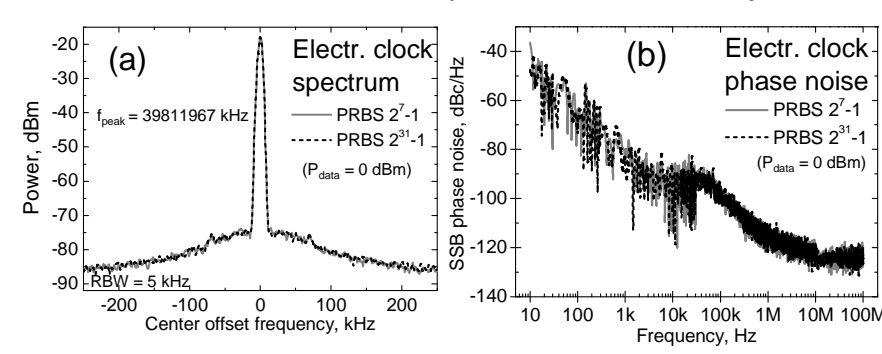

(c) Electrical clock: integrated jitter

\begin{tabular}{|c|c|c|}
\hline PRBS $/ \mathbf{P}_{\text {data }} \rightarrow$ SOA & 10Hz-100MHz & 100 Hz-10MHz \\
\hline $2^{7}-1 / 0 \mathrm{dBm}$ & $149 \mathrm{fs}$ & $75 \mathrm{fs}$ \\
\hline $2^{7}-1 /-3 \mathrm{dBm}$ & $139 \mathrm{fs}$ & $75 \mathrm{fs}$ \\
\hline $2^{7}-1 /-6 \mathrm{dBm}$ & $132 \mathrm{fs}$ & $73 \mathrm{fs}$ \\
\hline $2^{31}-1 / 0 \mathrm{dBm}$ & $132 \mathrm{fs}$ & $76 \mathrm{fs}$ \\
\hline $2^{31}-1 /-3 \mathrm{dBm}$ & $134 \mathrm{fs}$ & $75 \mathrm{fs}$ \\
\hline $2^{31}-1 /-6 \mathrm{dBm}$ & $147 \mathrm{fs}$ & $77 \mathrm{fs}$ \\
\hline
\end{tabular}

(d) $40 \mathrm{GHz}$ optical clock

Fig. 3. Recovered $40 \mathrm{GHz}$ clocks in $640 \mathrm{Gbit} / \mathrm{s}$ lab-trial. (a) Spectrum of recovered electrical clock, (b) Single side-band phase noise measurements on electrical clock when $\mathrm{P}_{\text {data }}=0 \mathrm{dBm}$, (c) Timing jitter values obtained by integrating the SSB phase noise over the intervals $10 \mathrm{~Hz}-100 \mathrm{MHz}$ and $100 \mathrm{~Hz}-10 \mathrm{MHz}$ for short/long PRBS and different data input powers to SOA, (d) Optical clock (MLFL-2 pulses).

Fig. 3 shows the spectrum (a), single side-band (SSB) phase noise measurements (b), and timing jitter (c) of the recovered electrical clock from the Gunn oscillator, as well as the optical clock pulses from the MLFL-2 (d). The SSB phase noise of the electrical clock was measured for PRBS $2^{7}-1$ and $2^{31}-1$ data patterns. Furthermore, the dynamic range of the CR circuit was investigated by varying the data input power to the SOA over a $6 \mathrm{~dB}$ range, from $\mathrm{P}_{\text {data }}=0 \mathrm{dBm}$ to $-6 \mathrm{dBm}$ (both for long 


\section{OWS2.pdf}

and short PRBS). Fig. 3 (a) and (b) show the spectrum and phase noise, respectively, of the recovered electrical clock in the case of $\mathrm{P}_{\mathrm{data}}=0 \mathrm{dBm}$. Integration of the measured SSB phase-noise spectra over the ranges $10 \mathrm{~Hz}-100 \mathrm{MHz}$ and $100 \mathrm{~Hz}-10 \mathrm{MHz}$, yields the timing jitter values shown in Fig 3 (c). These reveal the low power requirement of the CR circuit since the electrical clock jitter remains low ( $<150 \mathrm{fs}$ ) for data powers down to $-6 \mathrm{dBm}$, both for long and short PRBS patterns. As another advantage, the optical clock pulses obtained from the circuit (see Fig. 3 (d)), can be used directly for demultiplexing, i.e. in another SOA [4]. The circuit has the potential for integration, since the MLFL-2 can in principle be replaced by i.e. a semi-conductor based pulse source.

A field-trial was also conducted in order to test the performance of the CR circuit in the presence of transmission-induced phase fluctuations of the incoming data signal. The experimental set-up is shown in Fig. 1. The field-installed fibre is located around the city of Eindhoven in the Netherlands, and consists of $54.3 \mathrm{~km}$ of standard SMF. The dispersion in the link was post-compensated by using appropriate lengths of dispersion-compensating fibre. Due to insufficient dispersion-slope compensation, it was not possible to transmit the $640 \mathrm{Gbit} / \mathrm{s}$ data, since the pulse broadening after the link was too large. The data rate was therefore lowered to $320 \mathrm{Gbit} / \mathrm{s}$, and the data pulses before/after transmission are shown in Fig. 4 (a). Compared to the $640 \mathrm{Gbit} / \mathrm{s}$ lab-trial, there are a few minor changes in the set-up. Due to the different dispersion profile of the field-link, the wavelength allocation of MLFL- 1 and -2 are changed to $\lambda_{1}=1542 \mathrm{~nm}$ and $\lambda_{2}=1563 \mathrm{~nm}$. The last filter in the compression stage is a $5 \mathrm{~nm}$ BPF, resulting in broader $\sim 1$ ps pulses. At last, the data input power to the SOA for sufficient gain modulation is $\mathrm{P}_{\text {data }} \sim 6 \mathrm{dBm}$, which is attributed to an asymmetry in the SOA spectral gain-profile and the broader data-pulses (lower peakpower). The results of the field-trial are shown in Fig. 4 (b-e). The $40 \mathrm{GHz}$ clock is successfully recovered from the $320 \mathrm{Gbit} / \mathrm{s}$ transmitted data, both for PRBS $2^{7}-1$ and $2^{31}-1$. The spectrum and SSB phase noise of the recovered electrical clock are shown in Fig. 4 (b) and (c), respectively. Timing jitter values obtained by phase noise integration are shown in Fig. 4 (d), measured for short/long PRBS and $\mathrm{P}_{\mathrm{data}}=6 \mathrm{dBm}$ and $3 \mathrm{dBm}$. After transmission over the field-installed link, the $\mathrm{CR}$ circuit is able to recover the clock with a $10 \mathrm{~Hz}-100 \mathrm{MHz}$ timing jitter lower than $200 \mathrm{fs}$ (for $\mathrm{P}_{\text {data }}>3 \mathrm{dBm}$ ).
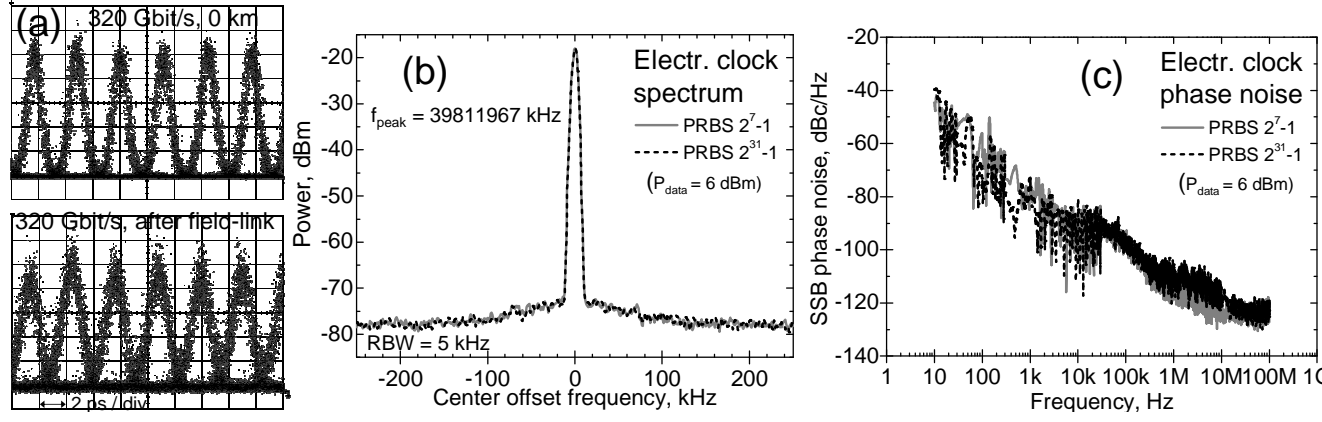

(d) Electr. Clock: integrated jitter \begin{tabular}{|l|c|c|}
\hline PRBS $/ \mathbf{P}_{\text {data }} \rightarrow$ SOA & $\mathbf{1 0 H z}-100 \mathrm{MHz}$ & $\mathbf{1 0 0 \mathrm { Hz } - 1 0 \mathrm { MHz }}$ \\
\hline
\end{tabular} \begin{tabular}{|l|c|c|}
\hline $2^{7}-1 / 6 \mathrm{dBm}$ & $154 \mathrm{fs}$ & $78 \mathrm{fs}$ \\
\hline $2^{7}-1 / 3 \mathrm{dBm}$ & $171 \mathrm{fs}$ & 100 fs \\
\hline
\end{tabular} \begin{tabular}{|c|c|c|}
\hline $2^{7}-1 / 3 \mathrm{dBm}$ & $171 \mathrm{fs}$ & $100 \mathrm{fs}$ \\
\hline $2^{31}-1 / 6 \mathrm{dBm}$ & $162 \mathrm{fs}$ & $96 \mathrm{fs}$ \\
\hline $2^{31}-1 / 3 \mathrm{dBm}$ & $231 \mathrm{fs}$ & $98 \mathrm{fs}$ \\
\hline
\end{tabular}

(e) $40 \mathrm{GHz}$ Optical clock

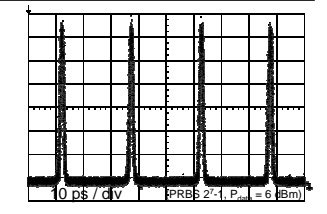

Fig. 4. Field-trial. (a) 320 Gbit/s OTDM data before and after transmission, (b) Spectrum of recovered $40 \mathrm{GHz}$ electrical clock, (c) Single side-band phase noise measurements on the electrical clock, (d) Timing jitter of the electrical clock, (e) Recovered $40 \mathrm{GHz}$ optical clock.

\section{Conclusion}

We have demonstrated $40 \mathrm{GHz}$ baserate clock recovery in a $640 \mathrm{Gbit} / \mathrm{s}$ OTDM lab-transmission over $50 \mathrm{~km}$. We used a potentially integrable clock recovery circuit with a high jitter-performance and low power requirements. The circuit was an injection-locked loop with a SOA as optical phase comparator. The recovered clock maintained a low jitter ( $<150 \mathrm{fs})$ at data input powers as low as $-6 \mathrm{dBm}$. Furthermore, a field-transmission over $54.3 \mathrm{~km}$ of $320 \mathrm{Git} / \mathrm{s}$ data confirmed the capability of the clock recovery circuit to produce a low-jitter clock ( $<200 \mathrm{fs}$ ) in the presence of transmission-induced phase-fluctuations.

This work was funded by STW EET6491. OFS Fitel Denmark ApS is acknowledged for providing the DF-HNLF and SMF+IDF transmission fibre. Rob Smets (Alcatel-Lucent) is acknowledged for providing advice and support.

\section{References}

[1] O. Kamatani, and S. Kawanishi, "Prescaled timing extraction from $400 \mathrm{~Gb} / \mathrm{s}$ optical signal using a phase lock loop based on four-wave-mixing in a laser diode amplifier", Photonics Tech. Lett., 1996, 8, (8), 1094-1096.

[2] C. Boerner, V. Marembert, S. Ferber, C. Schubert, C. Schmidt-Langhorst, R. Ludwig, and H.G. Weber, "320 Gbit/s clock recovery with electro-optical PLL using a bidirectionally operated electroabsorption modulator as phase comparator", Optical Fiber Communication Conference 2005 , paper OTuO3.

[3] L.K. Oxenløwe, D. Zibar, M. Galili, A.T. Clausen, L.J. Christiansen, and P. Jeppesen, "Filtering-assisted cross-phase modulation in a semiconductor optical amplifier enabling $320 \mathrm{~Gb} / \mathrm{s}$ clock recovery", European Conference on Optical Communication 2005, 3, paper We3.5.5, pp. 485-486.

[4] E. Tangdiongga, Y. Liu, H. de Waardt, G.D. Khoe, A.M.J. Koonen, H.J.S. Dorren, X. Shu, I. Bennion, "All-optical demultiplexing of 640 to 40 Gb/s using filtered chirp of a semiconductor optical amplifier", Optics Letters, 32, (7), pp. 835-837, 2007.

[5] Y. Yang, C. Lou, H. Zhou, J. Wang, and Y. Gao, "Simple pulse compression scheme based on filtering self-phase modulation-broadened spectrum and its application in an optical time-division multiplexing system", Applied Optics, 2006, 45, 28, 7524-7528. 